\title{
Performance and Reliability Comparison of 1T-1R RRAM arrays with Amorphous and Polycrystalline $\mathrm{HfO}_{2}$
}

\author{
Alessandro Grossi*, Eduardo Perez ${ }^{\dagger}$, Cristian Zambelli*, Piero Olivo*, and Christian Wenger ${ }^{\dagger}$ \\ *Università degli Studi di Ferrara, Dip. di Ingegneria, ENDIF, Via G. Saragat 1, 44122 Ferrara, Italy \\ ${ }^{\dagger}$ IHP, Im Technologiepark 25, 15236 Frankfurt (Oder), Germany
}

E-mail: alessandro.grossi@unife.it

\begin{abstract}
In this work, a comparison between 1T-1R RRAM 4kbits arrays manufactured either with amorphous or polycrystalline $\mathrm{HfO}_{2}$ in terms of performance, reliability, Set/Reset operations energy requirements, intra-cell and inter-cell variability during 10k Set/Reset cycles is reported. Polycrystalline array shows higher current ratio, lower switching voltages, lower power consumption, minor endurance degradation and higher overall yield than amorphous array. The drawbacks are represented by the higher Forming voltage, the larger read current distribution after Forming and the higher Reset voltage dispersion.
\end{abstract}

\section{INTRODUCTION}

Resistive Random Access Memories (RRAM) technology gathered significant interest for several applications [1]-[3]. RRAM behavior is based on the possibility of electrically modifying the conductance of a Metal-Insulator-Metal (MIM) stack: the Set operation moves the cell in a low resistive state (LRS), whereas Reset brings the cell in a high resistive state (HRS) [4], [5]. To activate such a switching behavior, some technologies require a preliminary Forming operation [6]-[8].

The choice of a proper MIM technology for RRAM cells, exhibiting good uniformity and low switching voltages, is therefore a key issue for array structures fabrication and reliable electrical operation [9]. Such a process step is mandatory to bring this technology to a maturity level. In this work, a comparison between 1T-1R RRAM 4kbits arrays manufactured either with amorphous [5] or polycrystalline [10] $\mathrm{HfO}_{2}$ is performed. In amorphous $\mathrm{HfO}_{2}$ the conduction mainly occurs through a conductive filament created during the Forming operation with highly variable concentration of defects, whereas in polycristalline $\mathrm{HfO}_{2}$ the conduction occurs only through grain boundaries with a very low defect concentration. These differences in terms of conduction properties and defect concentrations translate into different switching properties [9], with several implications on inter-cell (variations between cells) and intra-cell (cycle-to-cycle variations of any given cell) variability. In this work a comparison in terms of performance, reliability, Set/Reset operations energy requirements, intracell and inter-cell variability during $10 \mathrm{k}$ endurance cycles is reported.

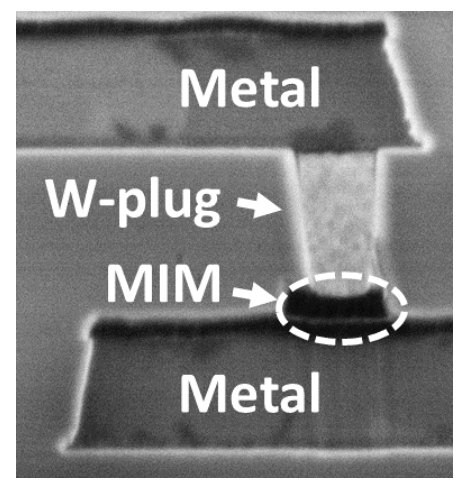

(a)

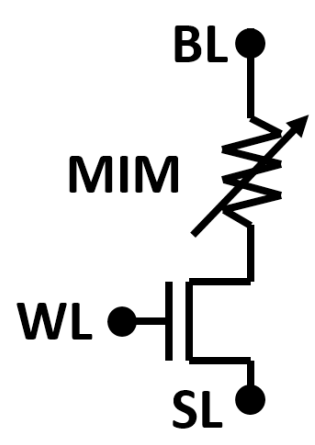

(b)
Fig. 1. Cross-sectional STEM image (a) and schematic (b) of the 1T-1R cell integrated in the arrays.

\section{EXPERIMENTAL SETUP}

The 1T-1R memory cells in the 4kbits arrays are constituted by a select NMOS transistor manufactured with a $0.25 \mu \mathrm{m}$ BiCMOS technology whose drain is in series to the MIM stack. The wordline (WL) voltage applied to the gate of the NMOS transistor allows setting the cell current compliance. The cross-sectional Scanning Transmission Electron Microscopy (STEM) image of the cell and the 1T-1R cell schematic are reported in Fig. 1. The variable MIM resistor is composed by $150 \mathrm{~nm}$ TiN top and bottom electrode layers deposited by magnetron sputtering, a $7 \mathrm{~nm}$ Ti layer, and a 8 $\mathrm{nm} \mathrm{HfO}_{2}$ layer deposited with two different Atomic Vapour Deposition (AVD) processes resulting either in amorphous (A) or polycrystalline (P) $\mathrm{HfO}_{2}$ films, respectively. The resistor area is equal to $0.4 \mu \mathrm{m}^{2}$. For amorphous films it has been integrated also a resistor with larger area that shows improved reliability and performance (i.e., $1 \mu \mathrm{m}^{2}$ ) [4]. The Forming/Set/Reset operations on the arrays were performed by using an Incremental Pulse and Verify algorithm [11]. The bitline (BL), sourceline (SL) and WL voltages applied during Forming, Set, Reset and Read operations are reported in Tab. I. Reset operations were performed by applying the WL voltage that allows maximizing the cells switching yield 
TABLE I

Forming, Set, Reset and Read Voltage Parameters.

\begin{tabular}{|l|c|c|c|}
\hline Operation & $\mathrm{V}_{S L}[\mathrm{~V}]$ & $\mathrm{V}_{B L}[\mathrm{~V}]$ & $\mathrm{V}_{W L}[\mathrm{~V}]$ \\
\hline Forming & 0 & $2-3.2$ & 1.5 \\
Set & 0 & $0.2-3.2$ & 1.5 \\
Reset & $0.2-3.2$ & 0 & $2.5(\mathrm{~A}) / 2.8(\mathrm{P})$ \\
Read & 0 & 0.2 & 1.5 \\
\hline
\end{tabular}

(2.8 $\mathrm{V}$ on array $\mathrm{A}$ and $2.5 \mathrm{~V}$ on array $\mathrm{P}$ ) while avoiding the breakdown of the $\mathrm{HfO}_{2}$ [12]. Pulses were applied during Forming by increasing $\mathrm{V}_{B L}$ with $\Delta \mathrm{V}_{B L}=0.01 \mathrm{~V}$, whereas during Set and Reset $\Delta \mathrm{V}_{B L}=0.1 \mathrm{~V}$ and $\Delta \mathrm{V}_{S L}=0.1 \mathrm{~V}$ have been used, respectively. Each pulse featured a duration of $10 \mu$ s, with a rise/fall time of $1 \mu \mathrm{s}$ to avoid overshoot issues. Set operation was stopped on a cell when the read-verify current reached at least $20 \mu \mathrm{A}$, whereas Reset was stopped when reached al least $10 \mu \mathrm{A}$. Forming, Set and Reset BL/SL voltages necessary to reach the requested read-verify current targets are extracted from the characterization data and labelled as $\mathrm{V}_{F O R M}, \mathrm{~V}_{S E T}$, and $\mathrm{V}_{R E S}$, respectively.

\section{EXPERIMENTAL RESULTS}

$1 \mathrm{~T}-1 \mathrm{R}$ cell arrays integrated with $\mathrm{A}-\mathrm{HfO}_{2}$ (A-array) with small $\left(0.4 \mu \mathrm{m}^{2}\right)$ and large $\left(1 \mu \mathrm{m}^{2}\right)$ resistor area, and P$\mathrm{HfO}_{2}$ (P-array) resulted in a Forming Yield (calculated as the cell percentage having a read verify current after forming $\mathrm{I}_{\text {read }} \geq 20 \mu \mathrm{A}$ ) of $58 \%, 90 \%$, and $95 \%$, respectively. Fig. 2 shows the average current ratios between Low Resistive State (LRS) and High Resistive State (HRS) read currents $\left(\mathrm{I}_{L R S} / \mathrm{I}_{H R S}\right)$, calculated on the entire cells population during Set/Reset cycling, and their relative dispersion coefficient. The dispersion coefficient, defined as $\left(\sigma^{2} / \mu\right)$, has been used to evaluate the cell-to-cell variability. The minimum current ratio that allows to correcly discriminate between HRS and LRS $\left(\mathrm{I}_{L R S} / \mathbf{I}_{H R S}>2\right)$ is indicated for comparison purposes [5]. Due to the faster cell degradation, the average ratios of Aarrays with resistor area of $0.4 \mu \mathrm{m}^{2}$ and $1 \mu \mathrm{m}^{2}$ cross the minimum ratio limit after 200 and $1 \mathrm{k}$ cycles, respectively. P-array showed higher ratio $(\approx 2.8)$ even after $10 \mathrm{k}$ cycles, but also a higher dispersion coefficient after Forming (i.e., cycle 1). The grain boundaries conduction mechanism in the polycrystalline $\mathrm{HfO}_{2}$ structure could be the reason of the higher cell-to-cell variability in P-arrays [13]. A-array with resistor area of $1 \mu \mathrm{m}^{2}$ shows a slightly higher average ratio and a slower degradation than A-array with resistor area of $0.4 \mu \mathrm{m}^{2}$. In smaller cells the presence of defects in the $\mathrm{HfO}_{2}$ stack has a stronger impact on the performance since makes the switching operations more difficult to control, speeds up the degradation and increases the overall inter-cell variability [14].

Fig. 3 shows a comparison between $\mathrm{I}_{L R S}$ and $\mathrm{I}_{H R S}$ cumulative distributions measured at cycle 1 and after the $10 \mathrm{k}$ Set/Reset cycling test: A-arrays show more compact distributions at cycle 1 , however after cycling P-array shows a higher percentage of correctly switching cells reaching the Set/Reset verify targets. $\mathrm{I}_{H R S}$ cumulative distribution in P-array shows

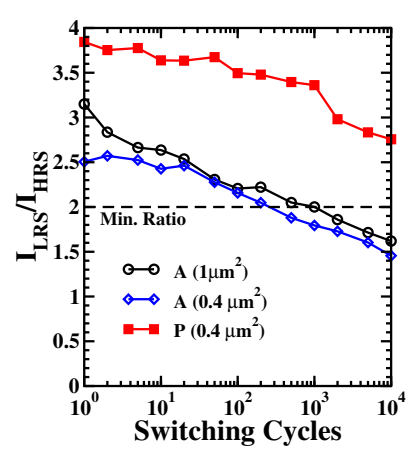

(a)

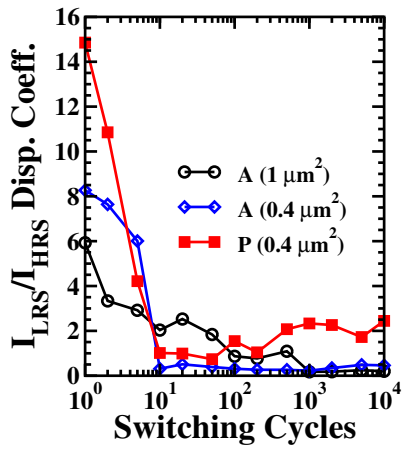

(b)
Fig. 2. $\mathrm{I}_{L R S} / \mathrm{I}_{H R S}$ current ratio average values (a) and dispersion coefficients (b) calculated during cycling.

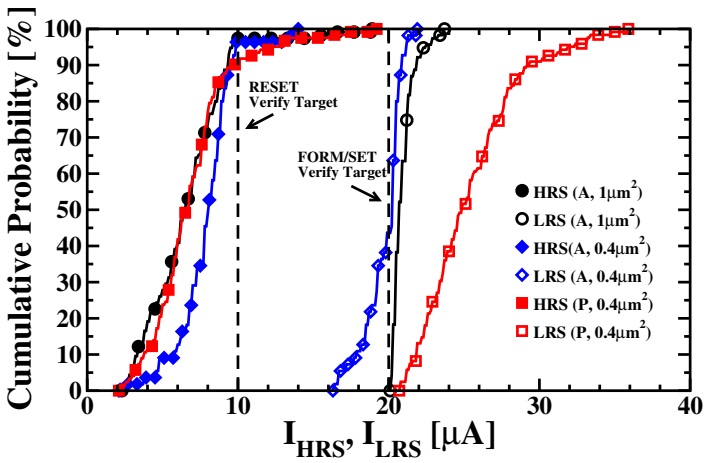

(a)

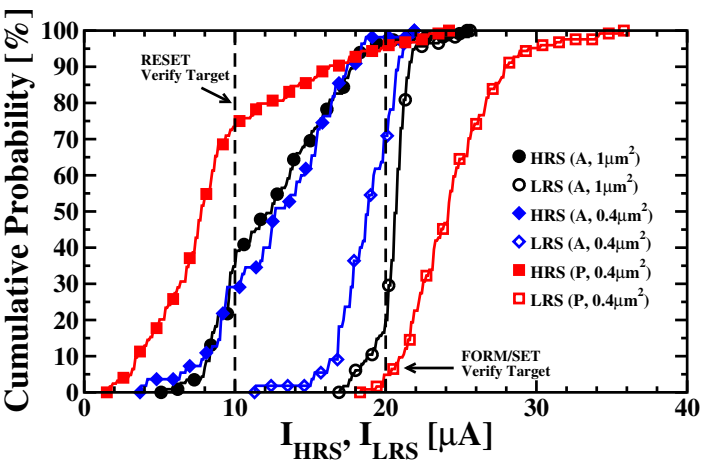

(b)

Fig. 3. $\mathrm{I}_{H R S}$ and $\mathrm{I}_{L R S}$ cumulative distributions at cycle 1 (a) and at cycle $10 \mathrm{k}$ (b).

a larger distribution tail at cycle 1 compared to A-arrays. After 10k cycles the cells degradation makes more difficult to break or re-create the filament, hence the voltage requested to reach the verify target increases as well as the number of cells not able to reach the verify target. An enlargement of the upper tail in P-array HRS distribution can be observed whereas on A-arrays a strong shift of the distributions towards higher currents occurs, since a higher number of cells is not able reach the Reset threshold. The reason of the lower ratio in A-array with small resistor area can be explained by the cumulative distributions, since they show lower $\mathrm{I}_{L R S}$ and higher $\mathrm{I}_{H R S}$ than cells with larger resistor area either at cycle 1 and after 


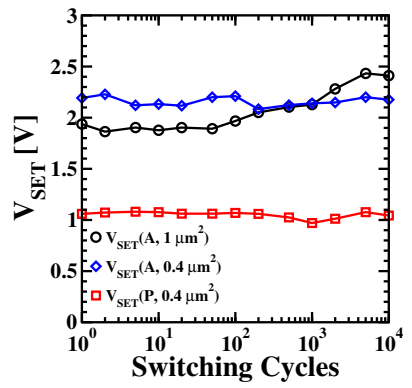

(a)

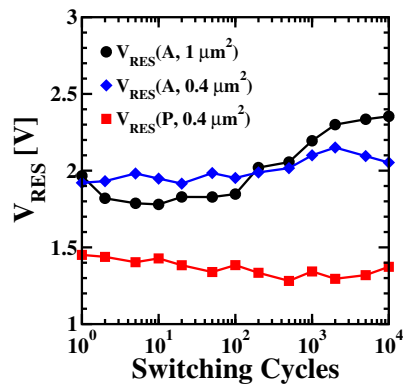

(c)

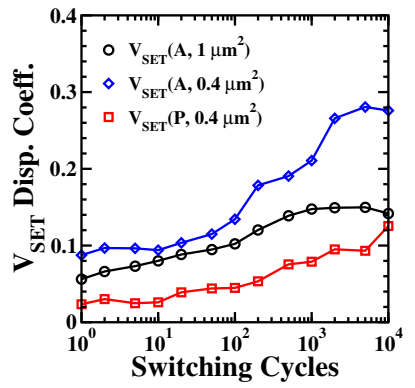

(b)

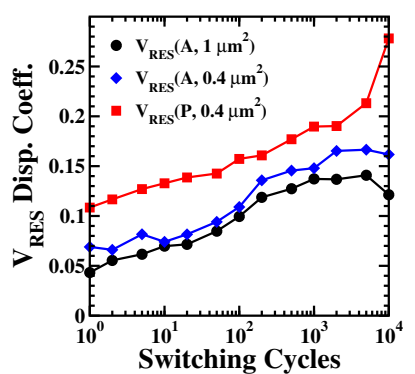

(d)
Fig. 4. $\mathrm{V}_{S E T}$ and $\mathrm{V}_{R E S}$ average values (a,c) and dispersion coefficients (b,d) calculated during cycling.

10k cycles. In $\mathrm{I}_{L R S}$ cumulative distributions the cells not able to reach the Set verify target generate a lower tail on P-arrays after 10k cycles, whereas on A-arrays a higher number of cells is not able to reach the Set verify target especially when cells with resistor area of $0.4 \mu \mathrm{m}^{2}$ are considered. This results into a strong shift of the distributions towards lower currents, especially in A-array with small resistor area that shows a high number of cells not reaching the Set verify target even at cycle 1 .

Fig. 4 shows the average Set and Reset switching voltages $\left(\mathrm{V}_{S E T}, \mathrm{~V}_{R E S}\right)$ and their relative dispersion coefficients: lower $\mathrm{V}_{S E T}$ and $\mathrm{V}_{R E S}$ are required on $\mathrm{P}$-array which also shows no variations during Set/Reset cycling, whereas $\mathrm{V}_{S E T}, \mathrm{~V}_{R E S}$ increase on A-arrays during cycling. $\mathrm{V}_{R E S}$ on P-array shows the highest variability: such operation is critical and very difficult to control in RRAM arrays since it strongly depends on how the filament is created: over Forming, as well as endurance degradation, can make the filament difficult to disrupt, increasing the $\mathrm{V}_{R E S}$ variability [11]. A-arrays show similar behavior of the average $\mathrm{V}_{S E T}$ and $\mathrm{V}_{R E S}$ (a lower average $\mathrm{V}_{S E T}$ is observed on A-array with larger resistor area only up to 500 cycles), while a higher $\mathrm{V}_{S E T}$ and $\mathrm{V}_{R E S}$ dispersion can be observed in A-array with smaller resistor area.

Fig. 5 shows the cumulative distributions of switching voltages during Forming, while Fig. 6 shows the Set and Reset switching voltages cumulative distributions at cycle 1 and after the Set/Reset cycling. Forming, Set and Reset incremental pulse algorithms starting point and last attempt are indicated, corresponding to the first and the last voltage pulse available in the incremental pulse and verify procedure [11]. P-array re-

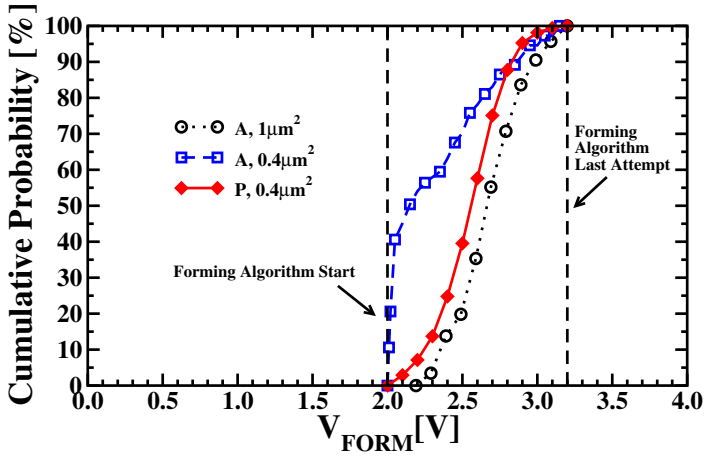

Fig. 5. $\mathrm{V}_{F O R M}$ cumulative distributions.

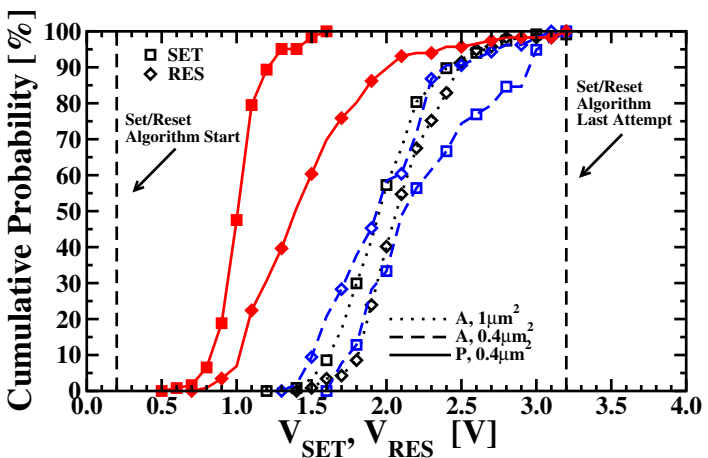

(a)

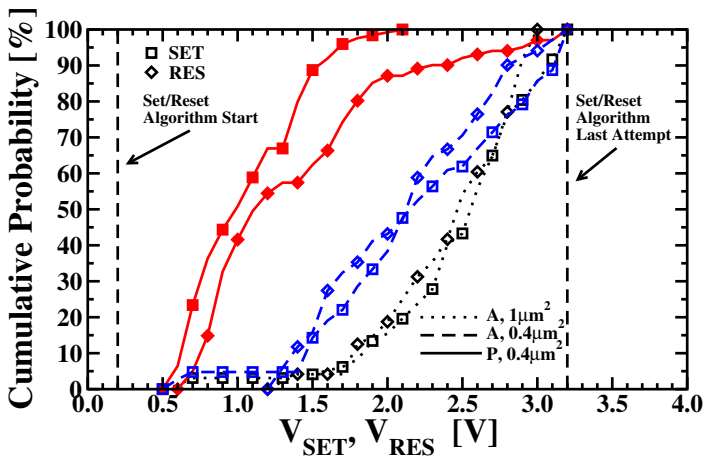

(b)

Fig. 6. $\mathrm{V}_{S E T}$ and $\mathrm{V}_{R E S}$ cumulative distributions at cycle 1 (a) and at cycle 10k (b).

quires lower $\mathrm{V}_{S E T}$ and $\mathrm{V}_{R E S}$ but higher $\mathrm{V}_{F O R M}$ if compared to A-array with the same resistor area. A-array with larger resistor area requires higher $\mathrm{V}_{F O R M}$. Moreover, it can be observed that $\approx 40 \%$ of the devices with smaller resistor area reached the forming threshold at $\mathrm{V}_{F O R M}=2 \mathrm{~V}$, corresponding to the first attempt of the Forming Algorithm. Since P-array shows a more compact distribution on $\mathrm{V}_{S E T}$ and a larger $\mathrm{V}_{R E S}$ than A-arrays, faster Set operation could be reliably used on P-array, whereas on Reset an incremental pulse with verify technique is required to ensure good reliability. A-arrays show large distributions on both $\mathrm{V}_{S E T}$ and $\mathrm{V}_{R E S}$, hence the adaptation of incremental pulse with verify techniques is mandatory on such arrays. 


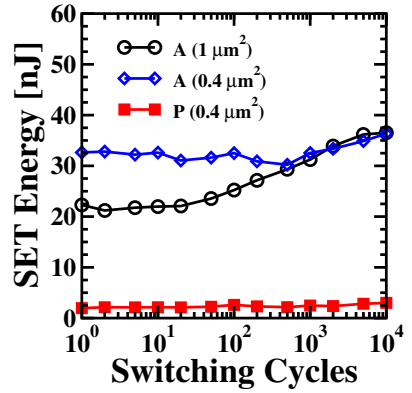

(a)

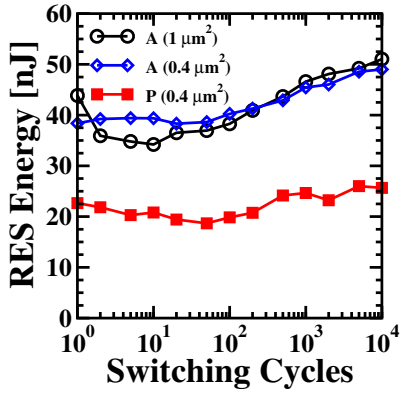

(b)
Fig. 7. Energy required to perform Set (a) and Reset (b) operations as a function of the Set/Reset cycle number.

Fig. 7 shows the average energy required to perform Set and Reset operations on a single cell. The overall energy required to create/disrupt the conductive filament during Set/Reset operations has been calculated as [11]:

$$
E=\sum_{i=1}^{n} V_{\text {pulse }, i} * I_{\text {pulse }, i} * T_{\text {pulse }}+V_{\text {read }} * I_{\text {read }, i} * T_{\text {read }}
$$

Where $n$ is the number of reset pulses applied during incremental pulse operation, $V_{\text {pulse, } i}$ is the pulse voltage applied at step $i, I_{\text {pulse }, i}$ is the current flowing through RRAM cell during pulse $i$ application, $T_{\text {pulse }}=10 \mu \mathrm{s}$ is the pulse length, $V_{\text {read }}=0.2 \mathrm{~V}$ is the read voltage applied during verify operation, $I_{\text {read }, i}$ is the current read on the RRAM during read verify step $i$, and $T_{\text {read }}=10 \mu s$ is the verify pulse length. Parray shows lower power consumption with a lower increase during cycling thanks to a lower $\mathrm{V}_{S E T}$ and $\mathrm{V}_{R E S}$. A-arrays with different resistor area show similar power consumption during Reset operation, whereas a lower consumption during Set is observed on A-array with larger resistor area only up to 500 cycles since cells with larger resistor area require lower $\mathrm{V}_{S E T}$.

\section{CONCLUSIONS}

1T-1R RRAM arrays manufactured with $\mathrm{P}-\mathrm{HfO}_{2}$ shows several advantages compared to $\mathrm{A}-\mathrm{HfO}_{2}$ even considering their improved process: higher current ratio, lower switching voltages, lower power consumption, minor endurance degradation and higher overall yield. Moreover, P-array show very low $\mathrm{V}_{S E T}$ variability, hence faster Set operation could be reliably performed. P-array disadvantages are represented by the larger HRS distribution after Forming, the higher Reset voltage dispersion and the higher $\mathrm{V}_{F O R M}$ if compared to A-array with the same resistor area, however but it must be pointed out that Forming operation is performed only once. The grain boundaries conduction mechanism in the polycrystalline $\mathrm{HfO}_{2}$ structure could be the reason of the higher cell-to-cell variability in P-arrays.

\section{ACKNOWLEDGMENTS}

This work was supported by the European Union's H2020 research and innovation programme under grant agreement $\mathrm{N}^{o}$ 640073.

\section{REFERENCES}

[1] S. Tanachutiwat, M. Liu, and W. Wang, "FPGA Based on Integration of CMOS and RRAM," IEEE Trans. on Very Large Scale Integration (VLSI) Systems, vol. 19, no. 11, pp. 2023-2032, Nov 2011.

[2] D. Garbin, E. Vianello, O. Bichler, Q. Rafhay, C. Gamrat, G. Ghibaudo, B. DeSalvo, and L. Perniola, "HfO2-Based OxRAM Devices as Synapses for Convolutional Neural Networks," IEEE Trans. on Electron Devices, vol. 62, no. 8, pp. 2494-2501, Aug 2015.

[3] T. Xu and V. Leppanen, "Analysing emerging memory technologies for big data and signal processing applications," in Digital Information Processing and Communications (ICDIPC), 2015 Fifth International Conference on, Oct 2015, pp. 104-109.

[4] C. Zambelli, A. Grossi, D. Walczyk, T. Bertaud, B. Tillack, T. Schroeder, V. Stikanov, P. Olivo, and C. Walczyk, "Statistical analysis of resistive switching characteristics in ReRAM test arrays," in IEEE Int. Conf. on Microelectronics Test Structures (ICMTS), Mar 2014, pp. 27-31.

[5] C. Zambelli, A. Grossi, P. Olivo, D. Walczyk, J. Dabrowski, B. Tillack, T. Schroeder, R. Kraemer, V. Stikanov, and C. Walczyk, "Electrical characterization of read window in ReRAM arrays under different SET/RESET cycling conditions," in IEEE Int. Memory Workshop (IMW), May 2014, pp. 1-4.

[6] D. Walczyk, T. Bertaud, M. Sowinska, M. Lukosius, M. A. Schubert, A. Fox, D. Wolansky, A. Scheit, M. Fraschke, G. Schoof, C. Wolf, R. Kraemer, B. Tillack, R. Korolevych, V. Stikanov, C. Wenger, T. Schroeder, and C. Walczyk, "Resistive switching behavior in TiN/HfO ${ }_{2} / \mathrm{Ti} / \mathrm{TiN}$ devices," in Int. Semiconductor Conf. DresdenGrenoble (ISCDG), Sept 2012, pp. 143-146.

[7] P. Lorenzi, R. Rao, and F. Irrera, "Forming kinetics in $\mathrm{HfO}_{2}$-based RRAM cells," IEEE Trans. on Electron Devices, vol. 60, no. 1, pp. 438-443, 2013.

[8] A. Grossi, D. Walczyk, C. Zambelli, E. Miranda, P. Olivo, V. Stikanov, A. Feriani, J. Sune, G. Schoof, R. Kraemer, B. Tillack, A. Fox, T. Schroeder, C. Wenger, and C. Walczyk, "Impact of Intercell and Intracell Variability on Forming and Switching Parameters in RRAM Arrays," IEEE Trans. on Electron Devices, vol. 62, no. 8, pp. 25022509, Aug 2015.

[9] K. Morgan, R. Huang, S. Pearce, and C. de Groot, "The effect of atomic layer deposition temperature on switching properties of $\mathrm{HfOx}$ resistive RAM devices," in IEEE Int. Symp. on Circuits and Systems (ISCAS), June 2014, pp. 432-435.

[10] H.-D. Kim, F. Crupi, M. Lukosius, A. Trusch, C. Walczyk, and C. Wenger, "Resistive switching characteristics of integrated polycrystalline hafnium oxide based one transistor and one resistor devices fabricated by atomic vapor deposition methods," Journal of Vacuum Science and Technology B, vol. 33, no. 5, pp. 052 204.1-052 204.5, 2015.

[11] A. Grossi, C. Zambelli, P. Olivo, E. Miranda, V. Stikanov, C. Walczyk, and C. Wenger, "Electrical characterization and modeling of pulse-based forming techniques in RRAM arrays," Solid-State Electronics, vol. 115, Part A, pp. 17 - 25, 2016. [Online]. Available: http://www.sciencedirect.com/science/article/pii/S0038110115002828

[12] A. Grossi, C. Zambelli, P. Olivo, E. Miranda, V. Stikanov, T. Schroeder, C. Walczyk, and C. Wenger, "Relationship among current fluctuations during forming, cell-to-cell variability and reliability in RRAM arrays," in IEEE Int. Memory Workshop (IMW), May 2015, pp. 1-4.

[13] V. Iglesias, M. Porti, M. Nafra, X. Aymerich, P. Dudek, and G. Bersuker, "Dielectric breakdown in polycrystalline hafnium oxide gate dielectrics investigated by conductive atomic force microscopy," Journal of Vacuum Science and Technology B, vol. 29, no. 1, 2011.

[14] S. Balatti, S. Ambrogio, A. Cubeta, A. Calderoni, N. Ramaswamy, and D. Ielmini, "Voltage-dependent random telegraph noise (RTN) in HfOx resistive RAM," in IEEE Int. Reliability Physics Symposium, June 2014, pp. MY.4.1-MY.4.6. 